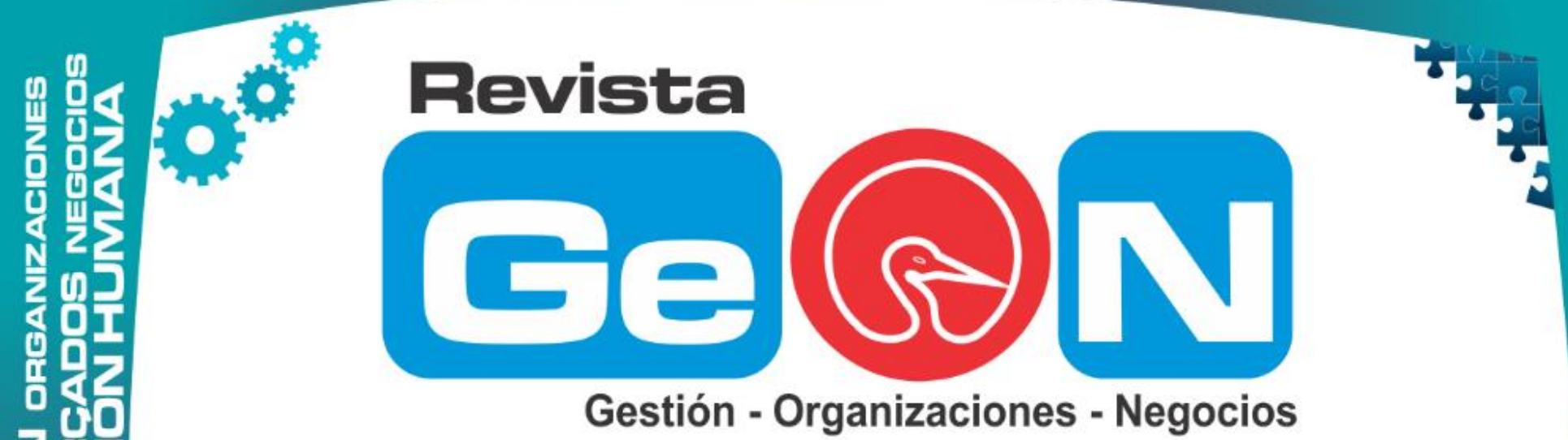

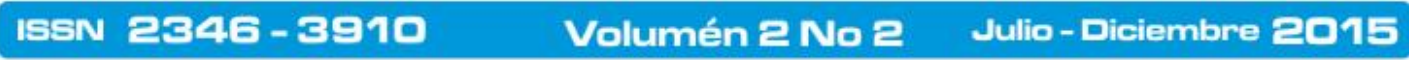

Revista Electrónica de la Facultad de Ciencias Económicas de la Universidad de los Llanos

1. 3 II

440

VILLAVICENCIO - COLOMBIA

$>2$

64

2

2 平品

70

ব)

展

o

ii III

0 U

ใด

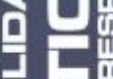

居的

임

Iiin in

照

只

造

iil 2

if in

4 吕

2

400

付 iI
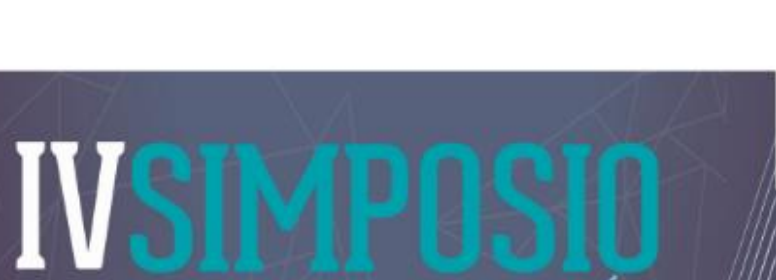

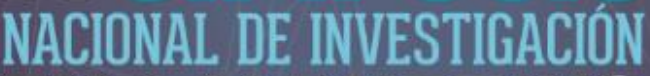

EN MARKETING
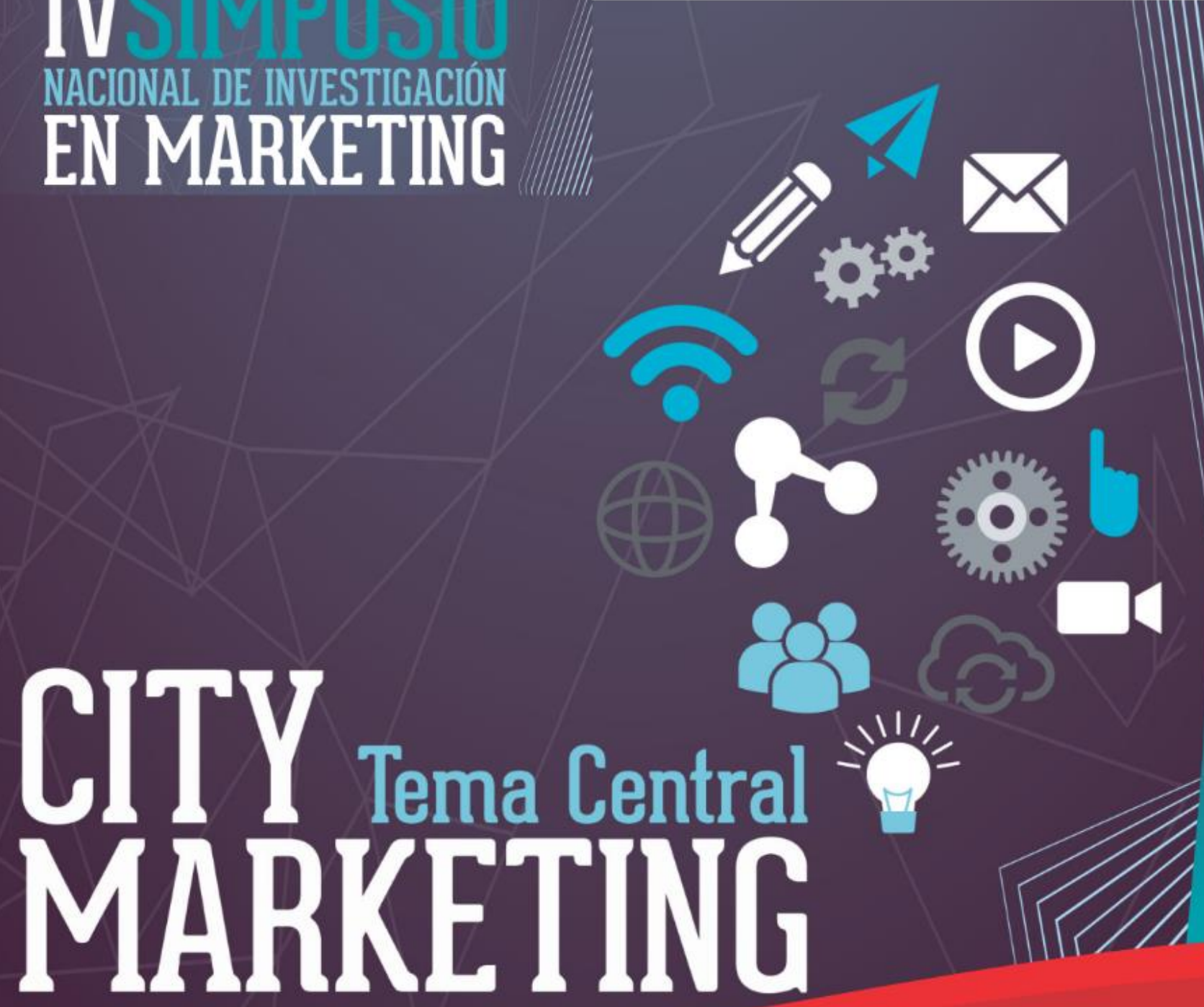

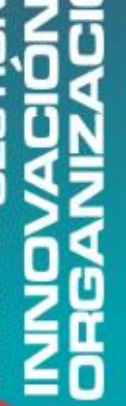
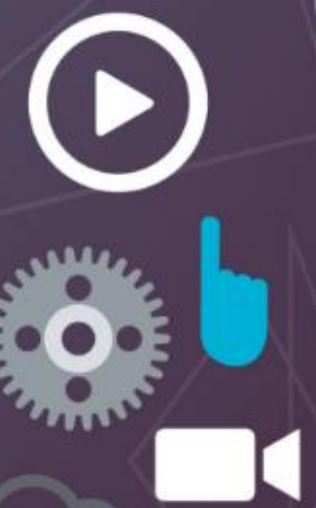


\section{Estrategias comerciales para la competitividad en una ciudad intermedia: caso Manizales - Rocio Tabares - Diego López}

Rocío Tabares Hoyos

Magister en Desarrollo Educativo y Social y

Especialista en Gerencia de Mercadeo

roci@umanizales.edu.co

Diego López Cardona,

Ph.D Ingeniería Informática, Magister

Docencia. Educación.

dlopez@umanizales.edu.co.

\section{Resumen}

El presente artículo resulta del análisis de información recolectada desde el observatorio del comercio, con los comerciantes de la ciudad de Manizales. Se pretende mostrar unas características del sector comercio como actividad económica importante que aporta al PIB de la ciudad y que contribuye para el desarrollo social y económico de los habitantes; características que identifican a Manizales (ciudad intermedia) con un mercado en donde se aplica una gama de estrategias que están de acuerdo a las tendencias y que le dan condiciones de competitividad a sus empresas.

Conceptualmente se abordan generalidades de la competitividad del comercio, estrategias de venta para ser competitivo, estrategias promocionales, la competitividad del sector comercio y estrategias comerciales más utilizadas por los comerciantes. Se halla que los comerciantes Manizaleños utilizan una variedad de tácticas y acciones de promoción y de servicio al cliente las cuales van a tono con el marketing directo y el marketing relacional, las cuales están encaminadas a mantener cautivados a los clientes, demostrando que la planeación es necesaria para tener éxito y ser competitivos en lo que desarrollan en sus empresas, mostrando su capacidad y creatividad para atender al público objetivo. De igual manera éstas van dirigidas a cumplir los objetivos corporativos previstos con el fin de lograr dinamismo incrementar su cuota de participación en el mercado y por supuesto, enfrentar la competencia y superar la crisis económica que ha golpeado tanto la actividad comercial.

\section{Palabras clave}

Manizales; comercio; estrategias; promoción; descuentos; comerciantes; negocios; compradores; consumidores; demanda.

\section{Abstract \\ This article is the analysis of information collected from the observatory trade with merchants of the city of Manizales. We intend to show a pattern of trade industry as an important economic activity that contributes to the GDP of the city and contributing to the social and economic development of the people; identifying characteristics of Manizales (intermediate city) with a market where a range of strategies that are based on trends and conditions that give their companies competitiveness applies.}

Conceptually overview of trade competitiveness, sales strategies to be competitive, promotional strategies, competitiveness in commerce and trade strategies used by traders are addressed. It is that the Manizales traders use a variety of tactics and promotional and customer service which are in tune with direct marketing and relationship marketing, which are aimed at maintaining captivated customers, showing that planning is needed to succeed and be competitive in developing their businesses, demonstrating their ability and creativity to meet the target audience. In like manner they are aimed at meeting the planned corporate objectives in order to achieve dynamic increase its share in the market and of course, face competition and overcome the economic crisis which has hit both the business. 


\section{Keywords}

Manizales; commerce; strategies; promotion; discounts; retailers; businesses; buyers; consumers; demand.

\section{Introducción}

El programa de Mercadeo Nacional e Internacional de la Universidad de Manizales junto con FENALCO capítulo Manizales, está realizando el observatorio del comercio de la ciudad, como un ejercicio permanente de recolección de información a través de cuestionarios que se le formulan a los comerciantes y administradores de los diferentes negocios comerciales y de los distintos sectores del comercio, según sus bases de datos.

Este escrito es resultado de la exploración de los datos con lo cual se ha podido establecer los elementos $y$ factores que a nivel local determinan el comportamiento de esta actividad económica en la ciudad. Con base en parte de la información recolectada, se presenta el análisis de varios de los tópicos que comprende el observatorio del comercio en general, resultados que han orientado el diseño de algunas estrategias comerciales. En este escrito no se presenta el anàlisis por subsectores si no en general, por subsectores es otro documento. Unas de las temáticas contempladas son la competitividad del sector comercio y las estrategias comerciales para ser competitivo, que dan cuenta de cómo son las estrategias de competitividad empleadas por los comerciantes Manizaleños.

La importancia del observatorio radica en que se está analizando un sector económico de relevancia, como es el comercio, de gran impacto para la economía local porque es fuente de importantes ingresos económicos para la ciudadanía y para la economía en general, dado que el comercio es la acción que complementa la actividad de la producción y hace posible que el aparato productivo siga funcionando al permitir que los consumidores se puedan proveer de toda clase de mercancías pasando por diversos canales; a su vez, esta actividad económica promueve la demanda y la oferta de los demás productos de los diferentes sub sectores y de los múltiples segmentos que se encuentran en el mercado.

\section{Estrategias de venta para la Competitividad}

La competitividad es un aspecto que adquiere cada vez mayor relevancia en el campo empresarial, derivado de las nuevas exigencias de los contextos económicos actuales. La capacidad de gestión de una empresa, sus estrategias empresariales y la gestión de innovación, son elementos diferenciadores entre ellas; es decir, cada una de las destrezas empresariales que demuestre tener cada organización frente a sus competidores determinará su nivel y permanencia en el medio comercial, por su participación en el mercado y su rentabilidad, dimensiones básicas para la competitividad.

Desde lo planteado en (UNICENCIA, 2012) se puede definir la competitividad como una medida de la capacidad inmediata y futura de las empresas de diseñar, producir y vender bienes cuyos atributos en término de precios y más allá de los precios se combinan para formar un paquete más atractivo que el de productos similares ofrecidos por los competidores, esto significa que las razones diferenciales que una compañía formalice alcanzar en todas sus áreas, son las que establecerán su aptitud corporativa y mercantil en el entorno local, nacional o internacional; y si no se sabe manejar estas tácticas, toda la acción se vuelve en contra de los objetivos corporativos previstos.

Con base en los conceptos anteriores se puede inferir que la competitividad es el resultado de la interacción de diversos factores conectados por el dinamismo que demanda y por las facultades que enfrenta la actividad empresarial que condicionan su servicio y garantizan la aptitud y la posición de la empresa ante el cliente. "Proceso continuo de mejoramiento y de innovación que requiere objetivos precisos, amplios insumos (recursos naturales, humanos, capital, infraestructura), 
estrategias y un medio ambiente que permita adoptar con rapidez aquellas innovaciones que estén basadas en el conocimiento." (Cámara de comercio Medellín, 2008). Significa que no es un desarrollo estático, sino por el contrario es todo un conjunto de prácticas dinámicas que impulsan y respaldan la marcha empresarial, siendo el juez final que válida el progreso $\mathrm{y}$ despliegue de estas estrategias es el mercado.

Es en la venta "bien sea venta por mostrador, venta por el sistema de autoservicio o combinación de ambos sistemas" (Londoño y Navas, 2012), donde se hace efectivo el esfuerzo de la planeación y ejecución de las actividades, por tanto el comerciante no puede solo dedicarse a vender y comprar productos, implica que cada empresa pueda invertir en instrumentos y mecanismos que permitan acercar la mercancía al consumidor, empleando tácticas que lo atraigan y estimulen a realizar la compra, o a contratar a un experto en Marketing para que despliegue actividades para el logro de los objetivos que incluyan aumentar el conocimiento que el público tiene de la empresa y sus productos y pueda proporcionar, variedad, interés y estímulos, que lleven a promocionar las ventas.

Con relación a las ventas, la American Marketing Association, las define como el proceso personal o impersonal por el que el vendedor comprueba, activa y satisface las necesidades del comprador para el mutuo y continuo beneficio (del vendedor y el comprador), esto es posible con la determinación de las necesidades y/o deseos del comprador."La venta es una función que forma parte del proceso sistemático de la mercadotecnia y la puntualiza como toda actividad que genera en los clientes el último impulso hacia el intercambio" (Fischer y Espejo, 2004).

\section{Estrategias promocionales para activar ventas}

Es común escuchar que las empresas realizan permanentemente estrategias de promoción y descuentos en sus organizaciones con el fin impulsar las ventas de sus productos y lograr fidelización de sus clientes, con la intención de reactivar el comercio. Pero cuando las personas tanto comerciantes como compradores se refirieren a estos conceptos, se encuentra que hay confusión, porque muchos de ellos creen que las promociones sólo se refieren a rebajas del precio de los productos. En razón a esto, los comerciante afirman que a los consumidores sólo les gusta las promociones por la disminución en el costo de los productos que van a comprar.

La promoción es una de las variables del marketing mix, la cual comprende el desarrollo de diversas actividades que utilizan las organizaciones con el fin de promover los niveles de venta, es anunciar a través de varias formas y medios las razones de compra de sus productos; es decir, dan a conocer los atributos, cualidades y beneficios que estos ofrecen, por ello, los expertos dicen que son mecanismos de transmisión de información que se requiere planear, diseñar e implementar, buscando promover e incrementar las ventas, teniendo presente que no deben ser acciones aisladas si no que deben corresponder a planes y acciones comerciales. "La variedad y complejidad del plan variará según la empresa, la naturaleza de la misma, el producto y el mercado objetivo". (Rubio, 2009).

La filosofía del marketing mix se centra en la necesidad de mezclar o integrar distintas funciones, desarrollando simultáneamente líneas de trabajo en cada uno de los componentes; con ello aparecieron conceptos como el desnatado, penetración del producto, ciclo de vida del producto y las teorías de la determinación de precios, dando relevancia a las comunicaciones especialmente en la fuerza de venta y la publicidad; también tuvo relevancia la segmentación del mercado, el posicionamiento del producto y las relaciones con otras áreas funcionales de la organización (López, 2013, pág. 20).

La promoción es "el conjunto de técnicas integradas en el plan anual de marketing para 
alcanzar objetivos específicos a través de diferentes estímulos y acciones limitadas en el tiempo y en el espacio, orientadas a segmentos determinados",(Herrera, 2008); esto significa que son tácticas para animar en un periodo $\mathrm{y}$ sector determinando las compras pensadas desde las características del nicho al que van dirigidas, a la vez que son destrezas muy útiles para apoyar la publicidad e incentivar las ventas y extender la posición de las marcas en los consumidores del mercado acordado. Para esto se recurre a diferentes tácticas. El acierto y éxito de estas actividades dependen del ingenio, creatividad, conocimiento que se tenga del cliente y del presupuesto corporativo. "Tanto el desarrollo de la sociedad como el crecimiento económico se encuentran estrechamente ligados a la implementación constante de innovaciones tecnológicas" (López, 2013).

\section{Servicio al cliente como factor de competitividad}

El marketing se preocupa porque la categoría del servicio al cliente se convierta en la estrategia substancial de la empresa, pues no basta con tener un producto de alta calidad si en todo el proceso que rodea la entrega de éste, no hay un buen ambiente. El marketing relacional que busca captar y retener clientes, ha mostrado la relevancia de integrar todos los componentes de la venta de un producto con el fin de entregar y facturar los productos en forma efectiva, lo que permitirá alcanzar los objetivos de la empresa. En muchas organizaciones estas variables se han tratado en forma separada y sin relación, lo que ha resultado perjudicial para el posicionamiento en el medio empresarial.

El servicio es un eslabón clave en las relaciones de intercambio, por tanto debe ser efectivo para lograr la total satisfacción de los clientes y el mantenimiento de las relaciones a largo plazo permitiendo beneficios mutuos. No es solo éste sino también el Fulfillment ${ }^{8}$ que

\footnotetext{
${ }^{8}$ Fulfillment es la entrega rápida, completa y en buenas condiciones del pedido realizado por el cliente.
}

aunque no siempre se tiene en cuenta como condición necesaria, si es decisivo para que los clientes perciban que la empresa es seria en cada operación y movimiento comercial. Incluye tanto los distintos servicios ofrecidos (entrega a domicilio, llamada por teléfono gratuita, aceptación de varias tarjetas de crédito, prueba gratis), como el nivel de servicio (rapidez, exactitud, solución de problemas, aceptación de devoluciones y realización de cambios (Carnet L., 2004.Pag 15), variable que es parte del mercadeo directo.

El buen servicio al cliente tiene repercusiones importantes en los niveles de rentabilidad. Históricamente se ha concebido el servicio a la clientela desde una perspectiva muy restrictiva, esta sugiere que "la función principal es lograr que el producto correcto llegue al lugar correcto en el tiempo correcto" y se centra en los aspectos de distribución y logística, pero hoy en día ha surgido una nueva visión, en un contexto más amplio, con enfoques multidimensionales, que inciden en las relaciones que se establecen con los grupos objetivos específicos (Cristopher, Payne y Vallantyne, 2004).

\section{Metodología}

Los insumos de este escrito hacen parte de la información que se recolecta para el Observatorio del comercio de Manizales, el cual ha sido realizado como ejercicio exploratorio así: Fase Planeación, desde la información solicitada por las directivas de la FENALCO capítulo Manizales se hizo el instrumento de recolección de información, la encuesta. Fase Ejecución, cada mes de acuerdo con la base de empresas afiliadas, se aplicaba el cuestionario estructurado a 120 comerciantes de los diferentes subsectores del comercio de la ciudad. Fase Resultados, con el fin de determinar el comportamiento de ésta actividad económica y luego de la tabulación se procedió a realizar el análisis, la interpretación para la presentación y la comunicación de resultados. 


\section{Hallazgos}

\section{Estrategias comerciales más utilizadas que determinan la competitividad}

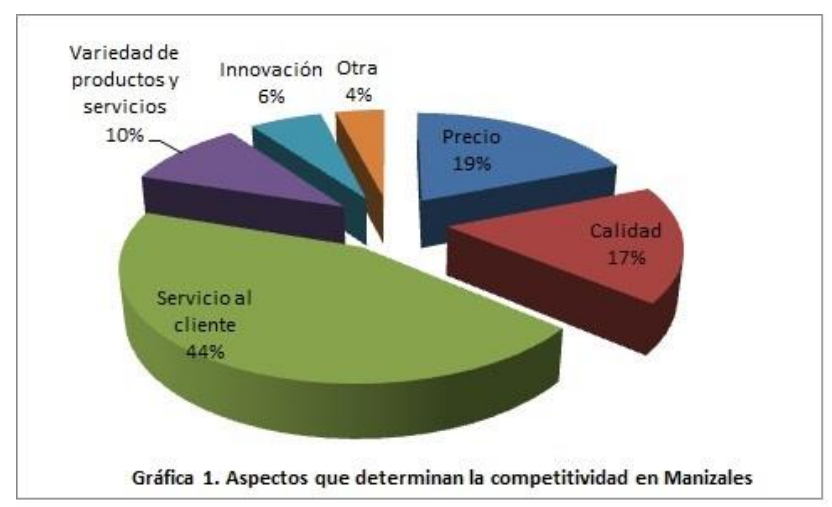

Los comerciantes de Manizales, para mantenerse competitivos en el mercado local, utilizan diferentes estrategias como publicidad, promociones, descuentos, bajos precios, tele mercadeo y atención al cliente; útiles para atraer compradores, mejorar la dinámica comercial de sus negocios y aumentar los niveles de venta; tácticas que según los expertos, son valiosas y apropiadas porque sirven para cautivar compradores, ya que en la medida que las personas conocen los productos, las promociones y actividades de la empresa acuden al negocio regularmente y esto genera compras.

De acuerdo a la gráfica 1, el $44 \%$ de los comerciantes entrevistados aseguran que el servicio al cliente los hace más competitivos por

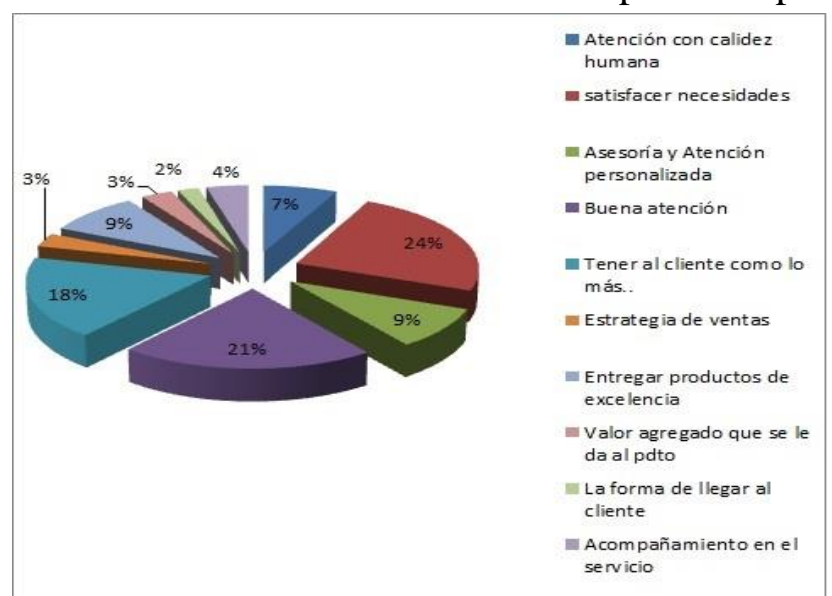

Gráfica 2. Tácticas para satisfacer al cliente

la atención que brindan al comprador; siguiendo en importancia el precio y luego la calidad, es decir, que estos tres factores (80\%) determinan la fidelidad del cliente, presumiendo que con la atención brindada y el precio pedido, adquieren un producto de calidad.

\section{Tácticas para brindar un buen servicio al cliente}

En la gráfica 2 se puede analizar diversas maneras que los comerciantes en general utilizan para brindar un buen servicio a sus clientes, entre las que se destacan: satisfacer las necesidades y expectativas $24 \%$, la buena atención $21 \%$, tener al cliente como lo más importante $18 \%$, lo que permite ver que el negociante tiene presente la importancia que representa el cliente para su empresa.

\section{Fidelización de los clientes}

Las mejores estrategias que consideran los comerciantes para fidelizar los clientes son el buen servicio (46\%) y los incentivos y promociones (21\%), como se observa en la gráfica 3. También tienen peso el contacto con el cliente (15\%) y el servicio posventa (15\%). Es importante resaltar que casi la totalidad de los comerciantes (97\%) busca fidelizar los clientes.

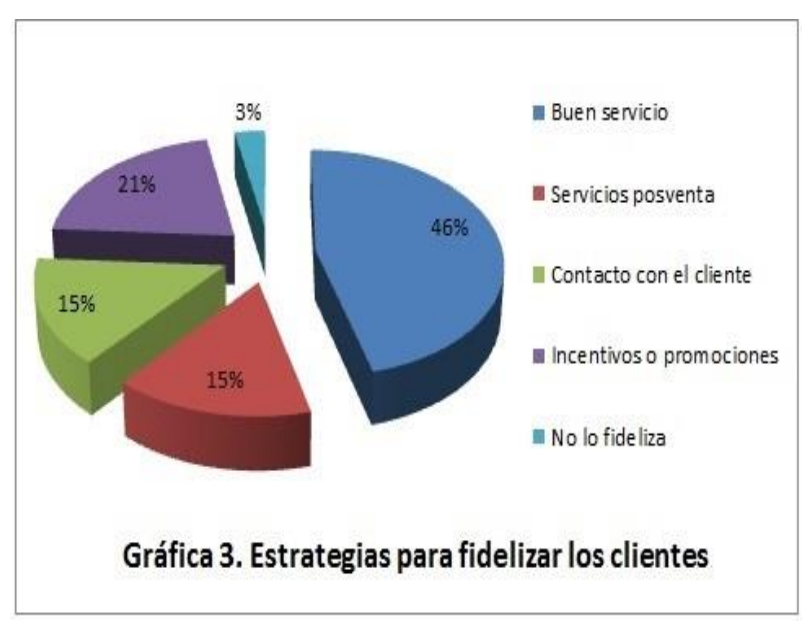

De acuerdo a la gráfica 4, la publicidad (27\%), las promociones $(24 \%)$ y los descuentos (17\%) son las estrategias más utilizadas para atraer clientes, mejorar la dinámica de sus negocios y aumentar los niveles de venta. 
La publicidad, estrategia de comunicación que presenta gran variedad de maneras de acercarse a los consumidores, no solo desde las formas tradicionales si no desde la pluralidad que hoy en día se ha consolidado a partir de la

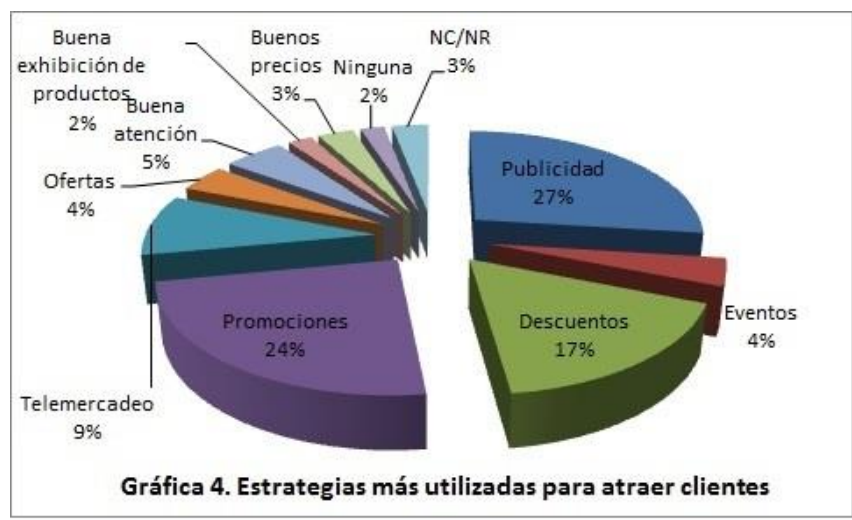

innovación tecnológica y los nuevos medios digitales. Por ejemplo, de gran auge es usar las redes sociales tales como" Facebook, Twitter o YouTube, sobre todo, si el público objetivo está conformado por un público joven. Crear una página en Facebook o abrir una cuenta en Twitter para captar seguidores, mantener comunicación con ellos; subir un video a YouTube en donde muestre actividades realizadas por la empresa y así promocionar los bienes o servicios" (Reyes y Gámez, 2013).

Entre las estrategias de promoción más utilizadas por los comerciantes para atraer clientes, predomina los descuentos (36\%), complementado con las ofertas (17\%), luego los bonos $(8 \%)$ y la publicidad (7\%). Las demás estrategias como rifas, premios, concursos no tienen mayor importancia. Vale mencionar que un porcentaje (14\%) no emplean ninguna actividad para atraer los clientes, como se muestra en la gráfica 5 .

\section{Problemas que afectan la dinámica comercial}

Como se observa en la gráfica 6 , más de la mitad de los comerciantes aseguran que la situación económica (32\%) y el desempleo $(21 \%)$ son los mayores factores que afectan la dinámica del comercio en general, factores inversamente proporcionales que de acuerdo a la mejora de uno se reduce el otro.

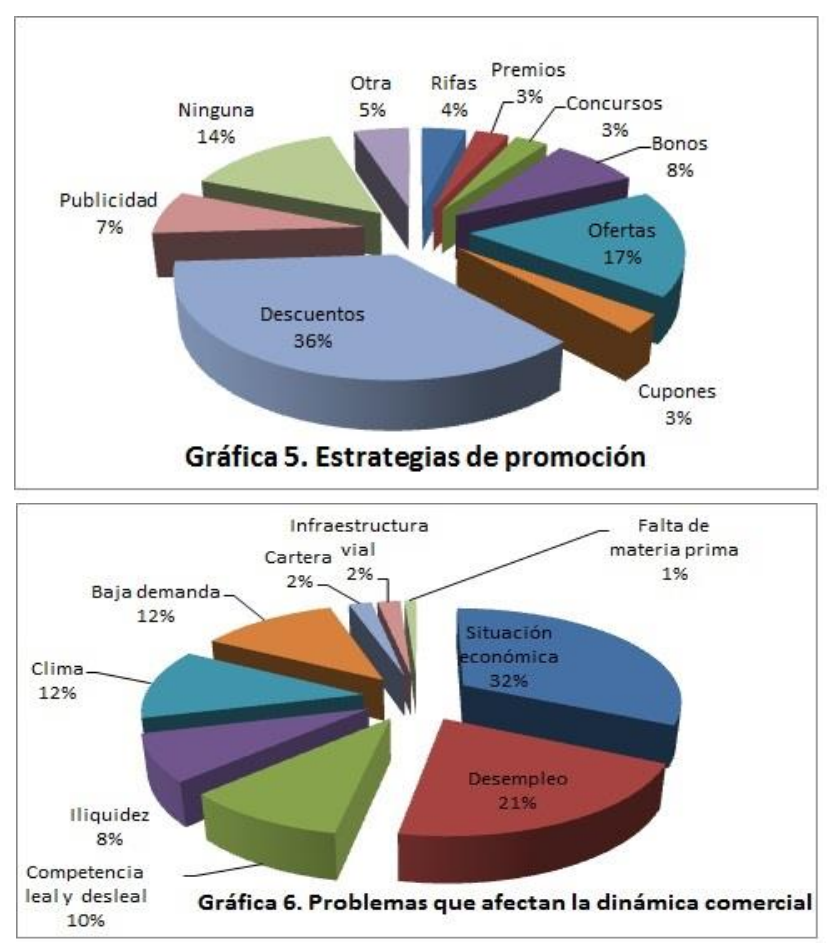

Los entrevistados consideran también otros factores que afectan la dinámica comercial, como la competencia leal y desleal (10\%), liquidez y falta de capital (8\%), clima (12\%) y baja demanda (12\%). Condicionantes de una buena actividad y estabilidad comercial.

Otra estrategia de aplicación por los empresarios en estos tiempos de crisis y competencia es tener buenos precios y/o bajos precios. Es una variable que se implementa en los diferentes almacenes, tiendas y centros comerciales de la ciudad. Lo hacen en unos meses específicos del año; es común ver cómo muchos negocios de la ciudad regularmente disminuyen el valor de las mercancías desde el $10 \%$ hasta el $50 \%$, con el fin de impulsar las ventas. Esta táctica trae consecuencias positivas para la empresa porque dinamiza y aumenta el volumen de las ventas.

\section{Conclusiones}

El comercio es importante como actividad mercantil impulsadora de posibilidades económicas para las empresas la cual se convierte en generadora de recursos económicos, permitiendo el crecimiento económico de las ciudades. 
Las estrategias más efectivas para la competitividad según los comerciantes entrevistados de la ciudad de Manizales son el buen servicio al cliente, la publicidad, las promociones y los descuentos y las menos efectivas son la exhibición de los productos, las ofertas, los eventos y la rebaja en precios.

Es relevante encontrar que el $44 \%$ de los comerciantes entrevistados consideran importante el servicio al cliente como estrategia para ser competitivos. Estrategia clave y necesaria si se quiere enfrentar la competencia, dada la dinámica comercial actual.

La buena atención como fuerte estrategia de venta que dicen tener los comerciantes manizaleños, es una táctica indispensable para mantener el buen nivel de ventas, condición de gran peso, en tanto que un cliente bien atendido es la mejor referencia para atraer otros más, y además, al estar satisfecho plenamente los demás indicadores para la compra no representan amenaza y lograr su lealtad.

Se deduce que la eficacia de las estrategias de venta implementadas dependerá del conocimiento y análisis que se tenga de las necesidades y expectativas del comprador, dado que el consumidor actual es más exigente $\mathrm{y}$ conocedor que el de hace unos años. Los empresarios y comerciantes pueden utilizar en sus organizaciones para mantener su grupo objetivo y un buen nivel de ventas, dirigir la promoción siempre al sujeto decisor de la compra.

Entre los problemas que más afectan el comercio en la ciudad se encuentran la situación económica y el desempleo, factores estructurales que afligen todos los sectores e impiden la buena dinámica comercial.

En estos tiempos de recesión y disminución de clientes son diversas las maneras que se pueden implementar para alcanzar el punto de equilibrio económico en las diferentes compañías, de ahí la variedad de tácticas que los empresarios ejecutan en sus firmas con el fin de contrarrestar o hacerle frente a los diferentes problemas que están afectando el comercio en general.

De las respuestas se puede inferir que hay una amplia y variada lista de tácticas que son poco utilizadas por los comerciantes e implementadas para la venta de sus productos, son una extensa gama de acciones que cada vez son más demandadas y valoradas por los consumidores como razones de compra. Son acciones que se pueden implementar para ser competitivo en la actividad comercial como: darle valor agregado al producto, variedad de productos, innovaciòn, bajar los precios, brindar servicios adicionales gratuitos, servicios posventa, darle al cliente experiencias agradables al momento de la compra, obsequios, incentivos, merchandising en el punto de venta, uso de redes sociales, contacto con el cliente, calidad de los productosentre otros.

\section{Referencias}

Amezcua Juan Bernardo (2012). "Mercadotecnia y Publicidad", sitio web de Slideshare, [en línea], disponible en: http://es.slideshare.net/bamezcua/5-promocinde-servicios-educativos, [accesado el día 05 de julio de 2014].

Cámara de Comercio de Medellín. (2008). "Informe Junta Directiva Cámara de Comercio 2008 de Medellín para Antioquia 2006-2008. Cluster. Una estrategia para crear ventaja competitiva", sitio web de Cámara de Comercio de Medellín, [en línea], disponible en: www.camaramedellin.com/estudioscamara.

Carnet Levi, Aray (2004). "Diseño de un modelo para elaborar actividades promocionales en Mercadeo Directo, que permitan maximizar la satisfacción en los consumidores meta, en empresas de consumo masivo. Caso práctico: Estrategias de mercadeo directo para tiendas de conveniencia BP", sitio web de Universidad Metropolitana, [en línea], disponible en: http://repositorios.unimet.edu.ve/docs/75/PGGM 2004A73L8.pdf. 
Chong José Luis (2007). "Promoción de venta, herramienta básica para el marketing integral" Ediciones Granica S. A .Buenos Aires, sitio web de Google Books, [en línea], disponible en: https://books.google.es/books?hl=es

Christopher Martin, Payne, y Adrian Vallantyne David. (2004). "Marketing relacional integrando lacalidad, el servicio al cliente y el marketing". Ediciones Díaz de santos.

Fischer, Laura y Espejo, Jorge. (2004). "Mercadotecnia" Mc Graw Hill, Págs. 31033.TerceraEdición.

Herrera Moreno, Andrés. C. (2008). "La importancia de la comunicación en la formación de marca para un artista de la música". Tesis Universidad Javeriana. Bogotá. Disponible enhttp://www.javeriana.edu.co/biblos/tesis/comu nicacion/tesis30.pdf.

Kotler y Armstrong. (2003). "Fundamentos de Marketing”, 6ta Edición, Prentice Hall, Págs. 470 y 476.

Londoño Aldana, Emperatriz y Navas Ríos, María Eugenia. (2012). "Estrategias de las Mypymes de Comercio: Análisis Basado en la Aplicación de las redes neuronales Artificiales", sitio web de Research Papers in Economics, [en línea], disponible en: http://ideas.repec.org/cgibin/htsearch?Q=estrateg ias+de+las+mypymes+de+comercio $\% 3 a+u n+a n$ $\%$ c3\%81lisis+basado+en+la+aplicaci\%c3\%93n+ de+las+redes+neuronales+artificiales, [accesado en agosto de 2014].
López Cardona, Diego (2013) “Ticcoffee: modelo de integración vertical en redes sociales para el comercio electrónico de café". Tesis Doctoral. Universidad Pontificia de Salamanca. Madrid, España.

López Raya, Agustín. (2013). "Producción e innovación tecnológica en el proceso de Digitalización de la RTVA". Tesis doctoral disponible, sitio web de Universidad de la Rioja, [en línea], disponible en: http://dialnet.unirioja.es/servlet/tesis?codigo $=38$ $\underline{812}$, [accesado en agosto de 2014].

Reyes y Gámez. (2013). Tesis. "Estrategias de mercadeo que permiten el incremento de las Ventas", sitio web de Biblioteca Virtual UJAP, [en línea], disponible en: http://bibliovirtualujap.files.wordpress.com/2013 /05/tesis-final11.pdf. , [accesado en julio de 2014]

Rubio Domínguez Pedro.2009. “¿Cómo llegar a ser un experto en marketing?", sitio web de Biblioteca virtual de Derecho, Economía y Ciencias Sociales, [en línea], disponible en: http://www.eumed.net/libros-gratis/2009a/506/ Tecnicas\%20de\%20Promocion\%20de\%20Venta s.htm.

UNICENCIA (2012). "Factores de competitividad aplicables en un estudio de caso de Uniciencia, ExtensiónBucaramanga" sitio web de Uniciencia, [en línea], disponible en: http://201.221.128.62:3000/Pagina/images/storie s/investigacion/TrabajoDefCompetitividad.pdf 